\title{
INFLUENCIA DE LAS ZONAS DE CRECIMIENTO EN LA COMPOSICIÓN FÍSICO QUÍMICA DEL FRUTO DE Solanum betaceum Cav.
}

\author{
Arturo A. Navarro Huaynates ${ }^{\mathrm{a}}$, Deysi Guzmán Loayza ${ }^{\mathrm{a}^{*}}$, Enrique Gonzales Mora ${ }^{\mathrm{a}}$
}

\section{RESUMEN}

En el presente estudio se evaluó el fruto del sacha tomate (Solanum betaceum Cav.) procedente de dos zonas de la región Cajamarca, los análisis realizados fueron: morfológico, de composición, proximal, perfil mineralógico y fitoquímico. Los resultados promedio de los análisis fueron: morfológico la proporción de pulpa $79,81 \%$, semilla $13,13 \%$, cáscara $6,22 \%$; el peso promedio del fruto fue de $75,61 \mathrm{~g}$; con respecto a las dimensiones del fruto se tiene: largo $65,23 \mathrm{~cm}$ y ancho $40,17 \mathrm{~cm}$; de acuerdo a método cielab el color del fruto es rojo amarillento; la dureza de la pulpa fue: $-1,75 \mathrm{Lb}$; contenido de humedad de $84,45 \%$; cenizas de $1,02 \%$, proteínas de $2,02 \%$, lípidos $0,065 \%$, fibra cruda de 8,03\%, extracto libres de nitrógeno de $4,37 \%$ y sólidos solubles de $9,83{ }^{\circ}$ Brix. Se identificaron metabolitos como: aminoácidos libres, taninos, flavonoides, leucoantocianidinas y alcaloides. El contenido promedio de polifenoles fue de $96,915 \mathrm{mg}$ EAG/100g de pulpa y el contenido promedio de flavonoides fue de 158,64 mg EQ/100 g de pulpa. En el perfil mineralógico se destaca la presencia de $\mathrm{Na}, \mathrm{K}, \mathrm{Ca}, \mathrm{Mg}$ y $\mathrm{P} ; \mathrm{Fe}, \mathrm{Cu}, \mathrm{Zn}, \mathrm{Mn}, \mathrm{B}$ y Si.

Palabras clave: Solanum betaceum, metabolitos secundarios, minerales, flavonoides, polifenoles.

\section{INFLUENCE OF GROWTH ZONES ON THE PHYSICAL CHEMICAL COMPOSITION OF THE FRUIT OF Solanum betaceum Cav.}

\begin{abstract}
In the present research, the fruit of the tomato sacha (Solanum betaceum Cav.) From two zones of the Cajamarca region was evaluated, a morphological, compositional, proximal, mineralogical and phytochemical profile was evaluated. The average results of the analyzes were: morphological the proportion of pulp $79,81 \%$, seed $13,13 \%$, husk $6,22 \%$; the average weight of the fruit was $75,61 \mathrm{~g}$; with respect to the dimensions of the fruit we have: length $65,23 \mathrm{~cm}$ and width 40,17 cm; According to cielab method the color of the fruit is yellowish
\end{abstract}

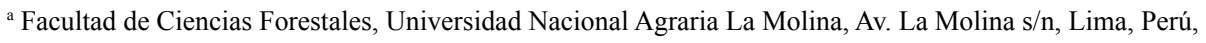
deysigl@lamolina.edu.
} 
red; the hardness of the pulp was: $-1,75 \mathrm{Lb} ; 84,45 \%$ moisture content; ash of $1,02 \%$, proteins of $2,02 \%$, lipids $0,065 \%$, crude fiber of $8,03 \%$, nitrogen-free extract of $4,37 \%$ and soluble solids of $9,83^{\circ}$ Brix. Metabolites were identified as: free amino acids, tannins, flavonoids, leucoanthocyanidins and alkaloids. The average content of polyphenols was $96,915 \mathrm{mg}$ EAG / $100 \mathrm{~g}$ of pulp and the average flavonoid content was $158.64 \mathrm{mg}$ EQ / $100 \mathrm{~g}$ of pulp. In the mineralogical profile the presence of $\mathrm{Na}, \mathrm{K}, \mathrm{Ca}, \mathrm{Mg}$ and $\mathrm{P}$ stands out; $\mathrm{Fe}, \mathrm{Cu}, \mathrm{Zn}, \mathrm{Mn}, \mathrm{B}$ and Si.

Key words: Solanum betaceum, secondary metabolites, minerals, flavonoid, polyphenols.

\section{INTRODUCCIÓN}

En la actualidad existe la necesidad de consumir o incluir en nuestra dieta diaria un porcentaje elevado de frutas y verduras, ya que estos alimentos nos favorecen con micronutrientes principalmente, tales como minerales, fibras y vitaminas además de compuestos polifenólicos ${ }^{1,2}$. Solanum betaceum Cav. de la familia Solanaceae, originaria de la vertiente oriental de los $\mathrm{Andes}^{3}$, comúnmente llamado sacha tomate, tomatillo en Perú, está ampliamente distribuido en nuestro territorio, esta especie crece entre los 1500 a 3000 msnm, sus frutos son consumidos en forma directa usándose en la preparación de diversos potajes, también tiene uso medicinal en el tratamiento de afecciones a la garganta, gripe, problemas hepáticos y control del colesterol ${ }^{2,3}$. Actualmente, existen muy pocos estudios reportados de este fruto en Perú, por lo general no indican la procedencia del mismo y por ello su composición varía dependiendo de la zona de origen ${ }^{4}$. En la presente investigación se planteó realizar la evaluación morfológica, nutricional, fitoquimica, cuantificar flavonoides y polifenoles y perfil mineralógico del fruto de Solanum betaceum Cav. para caracterizarlo en función de su procedencia.

\section{PARTE EXPERIMENTAL}

\section{Descripción de las zonas de estudio 5}

Zona 1: Distrito de Celendín, presenta temperatura mínima anual promedio de $9,9^{\circ} \mathrm{C}$, máxima anual promedio de $19,9^{\circ} \mathrm{C}$ y media anual promedio de $14,2^{\circ} \mathrm{C}$, la precipitación promedio total anual es de $986,41 \mathrm{~mm}$, su topografía es casi llana a fuertemente inclinada, con pendiente promedio de $2-20 \%$ sus suelos son de fertilidad media, los que pueden ser dedicados a actividades agrícolas y pecuarias, se encuentra ubicado dentro de la zona de vida conocida como Bosque húmedo-Montano bajo tropical (bh-MBT).

Zona 2: Distrito de Llacanora presenta una temperatura mínima anual promedio de $6,3^{\circ} \mathrm{C}$, máxima anual promedio de $20,8^{\circ} \mathrm{C}$ y media anual promedio de $13,6^{\circ} \mathrm{C}$ y la precipitación promedio total anual es de $499,40 \mathrm{~mm}$, presenta laderas inclinadas o muy empinadas, con pendientes entre 10 y $70 \%$; el suelo pertenece al grupo de leptosol, que son suelos 
desarrollados mayormente a partir de rocas areniscas cuarcíticas y en partes derivados de rocas calizas y volcánicas. La fertilidad natural es baja; con niveles bajos de materia orgánica, niveles medios a bajos en nitrógeno total, medios en fósforo disponible, medios a altos en potasio disponible y saturación de bases baja a media. Su aptitud se orienta a tierras de protección como refugio de la fauna silvestre, se encuentra ubicado dentro de la zona de vida conocida como Bosque seco-Montano bajo tropical (bs-MBT).

\section{Muestreo de los frutos}

Se obtuvieron $1,5 \mathrm{~kg}$ por árbol, seleccionándose frutos maduros, en buen estado físico y sanitario.

\section{Reactivos y equipos}

Todos los reactivos utilizados fueron calidad p.a. Los equipos utilizados fueron estufa eléctrica Heraeus (temperatura máxima $105 \pm 5^{\circ} \mathrm{C}$ ), balanza digital marca Ohaus de precisión 0,1mg; colorímetro Lovibond marca LC100 para la medida del color; penetrómetro Force Gauge modelo PCE-FM200 para la determinación de la dureza o firmeza, cuantificación de polifenoles y flavonoides totales, se utilizó el espectrofotómetro Helios $\gamma$, como estándares se utilizaron patrones de ácido gálico con $98 \%$ de pureza y quercetina con $95 \%$ de pureza, EtOH al $95 \%, \mathrm{AlCl}_{3}$ (p.a), acetato de potasio(p.a), para la cuantificación de minerales se utilizó el espectrofotómetro de plasma inducido (ICP), equipo micro Kjeldahl en la determinación de proteínas; para el análisis morfológico pie de rey, marca Vogel, sensibilidad $150 \times 0,01 \mathrm{~mm} / 6 \mathrm{x} 0,0005^{\prime}$.

\section{Métodos de análisis}

\section{Preparación de las muestras para los análisis}

Los frutos se pulpearon en forma manual, la pulpa obtenida se secó en estufa a $40^{\circ} \mathrm{C}$ por aproximadamente una semana, una vez secas se molió y tamizó para asegurar la homogeneidad de la muestra, se empacó en bolsas plásticas y se codificaron, luego se refrigeraron hasta su análisis.

Cuantificación de polifenoles, se siguió la metodología descrita por Romero $(2011)^{4}$, los resultados se expresaron como equivalentes de ácido gálico/100 g de pulpa (EAG).

Cuantificación de flavonoides, se siguió la técnica utilizada por Agudelo $(2013)^{6}$, utilizando como patrón quercetina, se preparó una solución madre, pesando $10 \mathrm{mg}$ del patrón $\mathrm{y}$ disolviéndolo en etanol al $80 \%$ hasta volumen final de $10 \mathrm{~mL}$ y a partir de ella se realizó una curva estándar con las siguientes concentraciones 12,$5 ; 25 ; 50$ y $100 \mathrm{mg} / \mathrm{L}$, obteniéndose un coeficiente de correlación de 0,9891 , para las muestras se pesaron 88 gramos de pulpa fresca y se le añadió $100 \mathrm{~mL}$ de etanol al $80 \%$, se sónico por $30 \mathrm{~min}$, se filtró en papel whatman $\mathrm{N}^{\circ} 44$ y se concentró en rotavapor hasta sequedad, luego, a partir de este extracto, se pesaron 
aproximadamente $0,11 \mathrm{~g}$ y se llevó a un volumen de $5 \mathrm{~mL}$ con una mezcla de $\mathrm{MeOH}: \mathrm{H} 2 \mathrm{O}$ (1:1); de esta solución se midieron $0,5 \mathrm{~mL}$ en un tubo de ensayo y se le agregaron los siguientes reactivos 1,5 $\mathrm{mL}$ de $\mathrm{EtOH}$ al $95 \%, 0,1 \mathrm{~mL}$ de $\mathrm{AlCl} 3(10 \%), 0,1 \mathrm{~mL}$ de acetato de potasio $1 \mathrm{M}, 2,8 \mathrm{~mL}$ de agua destilada, se reposó a temperatura ambiente por $30 \mathrm{~min}$, luego se leyó a $415 \mathrm{~nm}$, los resultados se expresaron como equivalentes de quercetina/ $100 \mathrm{~g}$ de pulpa (EQ).

\section{Análisis fisicoquímico}

Se utilizaron los métodos de la Association of Official Analytical Chemists AOAC (2007) ${ }^{\mathrm{T}}$ para el análisis proximal (determinación de humedad $\mathrm{N}^{\circ} 930.15$; determinación cenizas $\mathrm{N}^{\circ}$ 942.05; determinación de nitrógeno total $\mathrm{N}^{\circ}$ 955.04; determinación de fibra cruda $\mathrm{N}^{\circ}$ 962.09; determinación de extracto etéreo $\mathrm{N}^{\circ}$ 920.39).

\section{Análisis estadístico}

El diseño fue en dos bloques (correspondientes a las dos zonas geográficas) se trabajó por cada zona con tres individuos. Se utilizaron pruebas de Levene para ver la homogeneidad de las varianzas y también la prueba de Kolmogorov-Smirnov para ver la normalidad y prueba de T de Student para determinar si las medias son iguales. En todos los ensayos se realizaron cinco repeticiones.

\section{RESULTADOS Y DISCUSIÓN}

En la tabla 1 se presenta el análisis morfológico y observamos que la porción de pulpa de los frutos es alta de la zona 1, presentando mayor tamaño y ligeramente angostos a los reportados por Torres $(2012)^{8}$.

Tabla 1. Análisis morfológico del fruto de sacha tomate Solanum betaceum Cav.

\begin{tabular}{lcc}
\hline Propiedades físicas & Zona 1 & Zona 2 \\
\hline Cáscara $(\mathrm{g})$ & 4,02 & 6,14 \\
\hline Pulpa $(\mathrm{g})$ & 52,94 & 76,72 \\
\hline Semillas $(\mathrm{g}){ }^{\prime}$ & 6,37 & 16,21 \\
\hline Pedúnculo (g) & 0,53 & 9,93 \\
\hline Masa (g) & $65,72 \pm 6,58$ & $49,89 \pm 8,66$ \\
\hline Largo del fruto (mm) & $68,30 \pm 4,52$ & $62,17 \pm 4,83$ \\
\hline Ancho del fruto (mm) & $41,61 \pm 2,03$ & $38,74 \pm 2,27$ \\
\hline Cromaticidad L* & $47,93 \pm 3,22$ & $47,32 \pm 3,43$ \\
\hline Cromaticidad a* & $31,33 \pm 1,73$ & $27,85 \pm 2,59$ \\
\hline Cromaticidad b* & $32,47 \pm 2,27$ & $33,22 \pm 2,23$ \\
\hline Dureza (Lb) & $-1,66 \pm 0,36$ & $-1,84 \pm 0,27$ \\
\hline
\end{tabular}

'El valor incluye al tejido locular y placentario. 
Los frutos presentan coloraciones dentro de las tonalidades de rojo amarillentas, como lo demuestran los valores positivos de $\mathrm{a}^{*} \mathrm{y} \mathrm{b}^{* 9}$ para ambas zonas, el color de la cáscara está influenciada por los cambios con el estado de madurez de los frutos ${ }^{10}$, la diferencia de valores de $\mathrm{a}^{*}$ entre ambas zonas probablemente se deba a que los frutos de la zona 1 estén más expuestos a la radiación solar, provocando que la planta sintetice componentes coloreados que los de la zona $2^{11,12}$; en cuanto a la dureza o firmeza los valores negativos significan que los frutos estaban en buen estado, no se encontraban demasiado maduros, ya que estos frutos al no ser climatéricos se deben cosechar cerca de la madurez de consumo para obtener las mejores características organolépticas ${ }^{13}$.

En el tabla 2 se observa la composición química del fruto de sacha tomate, en este cuadro se compara con valores determinados en Colombia ${ }^{14}$, Venezuela ${ }^{8}$ y Perú $^{13}$ (lugar no determinado) el contenido de proteínas está dentro de los valores comparados, el de lípidos difieren mucho, siendo los más bajos de los cuatro y son los esperados ya que al tratarse de frutos estos no acumulan dichos componentes en sus tejidos; un fruto es apreciado por su contenido de fibra, por tanto los frutos de la zona 2 son los que presentan mayor contenido debido a la mayor presencia de tejido placentario rodeando a las semillas condicionando la forma, volumen, composición química y calidad de los frutos que al compararlos con los otros autores son ligeramente mayores a los reportados por Revelo $(2014)^{14}$. En cuanto al contenido de las sustancias minerales representados como cenizas son valores cercanos a los reportados por Torres $(2012)^{8}$ y Rojas $(2013)^{10}$, y estas representan el contenido mineral que el fruto ha almacenado hasta su cosecha.

Tabla 2. Composición química del fruto de Solanum betaceum Cav. por 100 gramos de porción comestible.

\begin{tabular}{|l|c|c|c|c|c|}
\hline \multirow{2}{*}{ Componente } & \multicolumn{5}{|c|}{ Contenido } \\
\cline { 2 - 6 } & $\begin{array}{c}\text { Revelo } \\
(\mathbf{2 0 1 4 )}\end{array}$ & $\begin{array}{c}\text { Torres } \\
\mathbf{( 2 0 1 2 )}\end{array}$ & $\begin{array}{c}\text { Rojas } \\
\mathbf{( 2 0 1 3 )}\end{array}$ & Zona 1 & Zona 2 \\
\hline Humedad (\%) & & & & $84,27 \pm 0,50$ & $84,64 \pm 0,16$ \\
\hline $\begin{array}{l}\text { Proteínas crudas } \\
(\%) \text { (factor 6,25) }\end{array}$ & $3,33 \pm 0,73$ & $1,78 \pm 0,14$ & $1,49 \pm 0,02$ & $2,27 \pm 0,03$ & $1,78 \pm 0,01$ \\
\hline Lípidos (\%) & $3,28 \pm 0,93$ & 0,16 & $0,34 \pm 0,01$ & $0,07 \pm 0,007$ & $0,06 \pm 0,006$ \\
\hline Fibras crudas (\%) & 5,47 & $4,10 \pm 0,00$ & $1,11 \pm 0,02$ & $6,19 \pm 0,09$ & $9,87 \pm 0,21$ \\
\hline Cenizas (\%) & $6,25 \pm 0,47$ & $0,88 \pm 0,01$ & $0,83 \pm 0,03$ & $1,03 \pm 0,009$ & $1,02 \pm 0,009$ \\
\hline ELN (\%)* & - & - & $7,50 \pm 0,15$ & $6,14 \pm 0,10$ & $2,60 \pm 0,21$ \\
\hline $\begin{array}{l}\text { Sólidos solubles } \\
{ }^{\circ} \text { Brix }\end{array}$ & $9,05 \pm 0,23$ & $10,51 \pm 1,20$ & $11,09 \pm 0,27$ & $10,17 \pm 0,89$ & $9,50 \pm 1,08$ \\
\hline
\end{tabular}

*ELN: Extracto libre de nitrógeno 
El detalle de la composición mineral se muestra en la tabla 3, donde observamos que destacan el fósforo, magnesio, potasio y calcio, los valores para estos elementos son bajos a los reportados por otros autores para la mayoría de los elementos identificados, pero en el caso del potasio se cuantificó $3510 \mathrm{mg} / \mathrm{kg}$ para la zona 1 y 4248,687 para la zona 2 y Torres $(2012)^{8}$ reporta $170 \mathrm{mg} / \mathrm{kg}$. Esta notoria diferencia en cuanto a la cantidad de los minerales se debería a que los suelos de la zona 1 tienen niveles medios de fósforo y contenidos elevados en potasio ${ }^{6}$, otra razón por la que un fruto tiende a almacenar mayor cantidad de un mineral que otro es la competencia que existe entre los minerales, así, en condiciones de exceso de potasio en el suelo, la planta incrementa el consumo de este elemento pudiendo interferir en la absorción y la disponibilidad de otros minerales como el calcio y magnesio.

Tabla 3. Perfil mineralógico del fruto de Solanum betaceum Cav.

\begin{tabular}{|c|c|c|}
\hline Minerales identificados & Zona 1 & Zona 2 \\
\hline Boro (mg/kg) (L.C: 0,25mg/kg) & 0,801 & 0,690 \\
\hline Sodio $(\mathrm{mg} / \mathrm{kg})$ (L.C: $1,5 \mathrm{mg} / \mathrm{kg})$ & 16,898 & 41,091 \\
\hline Magnesio (mg/kg) (L.C: 0,35mg/kg) & 150,957 & 123,322 \\
\hline Silicio $(\mathrm{mg} / \mathrm{kg})(\mathrm{L} . \mathrm{C}: 0,25 \mathrm{mg} / \mathrm{kg})$ & 1,802 & 4,411 \\
\hline Fósforo (mg/kg) (L.C: 0,5mg/kg) & 206,974 & 199,378 \\
\hline Potasio (mg/kg) (L.C: 4,5mg/kg) & 3510,939 & 4248,687 \\
\hline Calcio $(\mathrm{mg} / \mathrm{kg})(\mathrm{L} . \mathrm{C}: 2,5 \mathrm{mg} / \mathrm{kg})$ & 89,607 & 92,433 \\
\hline Manganeso (mg/kg) (L.C: 0,05mg/kg) & 0,791 & $<0,05$ \\
\hline Hierro (mg/kg) (L.C: 0,2mg/kg) & $<0,2$ & 0,924 \\
\hline Cobre $(\mathrm{mg} / \mathrm{kg})(\mathrm{L} . \mathrm{C}: 0,05 \mathrm{mg} / \mathrm{kg})$ & 0,527 & 0,525 \\
\hline Zinc $(\mathrm{mg} / \mathrm{kg})(\mathrm{L} . \mathrm{C}: 0,05 \mathrm{mg} / \mathrm{kg})$ & 0,879 & 0,661 \\
\hline
\end{tabular}

En el análisis elemental no se identificaron elementos pesados como plomo, cromo, cadmio y mercurio. Debemos destacar que el contenido de metabolitos primarios varía de una zona a otra, en donde se puede ver notablemente la influencia de las condiciones climáticas como las horas de luz y la precipitación pluvial en el desarrollo de los frutos.

En la tabla 4 se presentan los resultados de la evaluación fitoquimica y se observa la presencia de taninos, flavonoides, leucoantocianidinas, compuestos que conforman el grupo de los polifenoles, presencia que es similar a los reportados en frutos de S. betaceum de El Salvador ${ }^{15}$, en Solanum multifidum y Lycianthes lycioides, de Perú6,15; y en frutos de Solanum crinitipe; grupos que le estarían otorgando poder antioxidante a este fruto ya que esta característica depende de la concentración y de la naturaleza de los compuestos que están presentes en él. Estos resultados evidencian la presencia de flavonoides en la familia Solanaceae y además estarían contribuyendo a la coloración roja de la cáscara. 
Tabla 4. Identificación de los metabolitos secundarios

\begin{tabular}{lcc}
\hline \multicolumn{1}{c}{ Metabolitos } & Zona 1 & Zona 2 \\
\hline Taninos & + & + \\
\hline Flavonoides & + & + \\
\hline Aminoácidos libres & + & - \\
\hline Esteroides & - & - \\
\hline Quinonas & - & - \\
\hline Cardenólidos & - & - \\
\hline Alcaloides & + & + \\
\hline Luecoantocianidinas & + & + \\
\hline
\end{tabular}

Presencia: + no identificado: -

La familia de las solanáceas, especialmente las del género Solanum, son conocidas por sintetizar alcaloides ${ }^{15}$, con estos resultados positivos se comprueba su presencia, además observamos que no existe diferencia de la presencia entre la zona 1 y 2.

En base a estos resultados fitoquímicos se cuantificó los flavonoides y polifenoles, los resultados se muestran en la tabla 5 .

Tabla 5. Cuantificación de flavonoides y polifenoles en pulpa de

Solanum betaceum Cav.

\begin{tabular}{ccc}
\hline Metabolitos & Zona 1 & Zona 2 \\
\hline Flavonoides (mg EQ/100g pulpa) & $157,78 \pm 0,405$ & $159,50 \pm 0,168$ \\
\hline Polifenoles (mg EAG/100g pulpa) & $88,84 \pm 0,687$ & $104,99 \pm 0,586$ \\
\hline
\end{tabular}

\begin{abstract}
Algunos compuestos polifenólicos tienen un importante papel en la tolerancia al estrés en las plantas; la deficiencia de agua produce un aumento de los niveles de estos compuestos, como observamos en los valores de la zona 2 que es mucho mayor con respecto a los de la zona $1 \mathrm{e}$ igualmente podemos destacar la diferencia en el contenido de flavonoides entre ambas zonas, donde apreciamos la influencia de las condiciones climáticas, como bajas temperaturas, bajas precipitaciones y suelos con baja fertilidad, características que estarían influenciando en los valores presentados para la zona 2 .
\end{abstract}




\section{CONCLUSIONES}

El lugar donde se desarrolla una planta o un conjunto de plantas influye significativamente en su desarrollo físico y composición química, como lo demuestran los resultados obtenidos. Considerando la importancia de la presencia y contenido de polifenoles y flavonoides, los frutos de la zona 2 serían de mejor calidad, también podemos destacar que se podría obtener mayor producción de estos metabolitos estimulando a la planta bajo ciertas condiciones.

Finalmente, de acuerdo a las diferentes características de las zonas de estudio, los frutos de Solanum betaceum Cav. tienen un importante aporte nutricional, mineralógico, además de sustancias que cumplen una función importante como protectores contra el estrés oxidativo, tales como los polifenoles, flavonoides y leucoantocianidinas.

\section{AGRADECIMIENTOS}

Esta investigación se realizó en las instalaciones del Laboratorio de Pulpa y Papel del área de Transformación Química de la Madera de la Facultad de Ciencias Forestales de la UNALM. Al Técnico de laboratorio Luis E. Cabrera Vaquerizo por su apoyo en el desarrollo de esta investigación.

\section{REFRERENCIAS BIBLIOGRÁFICAS}

1. Kuskoski EM, Asuero AG, Troncoso AM, Mancini-Filho J, Fett R. Aplicación de diversos métodos químicos para determinar actividad antioxidante de pulpa de frutos. Ciênc. Tecnol. Aliment. Campinas [Internet]. 2005; 25(4): 726-732. [Citado 13 ago 2016] Disponible en: http://www.scielo.br/scielo.php?script=sci arttext\&pid=S0101-20612005000400016

2. Chávez CCA, Ortiz OKA. Efecto del consumo de jugo de tomate de árbol sobre indicadores bioquímicos en el personal administrativo de la Universidad Técnica del Norte, Ibarra. Periodo 2014-2015. [Tesis]. Ibarra: Universidad Técnica del Norte, Facultad de Ciencias de la Salud; 2016.

3. Cano LAP. Extracción y uso de tres pigmentos naturales a partir de tomate de árbol árbol (Solanum betaceum Cav.), mortiño (Vaccinium myttillus L.) y mora de castilla (Rubus glaucus) como alternativa colorante natural para alimentos. [Tesis]. Sangolquí: Escuela Politécnica del Ejército: Departamento de ciencias de la vida; 2011.

4. Romero PS, Domínguez TG, Guzmán LD. Cuantificación de polifenoles en hojas de un clon de Uncaria tomentosa (Will. Ex. Shult) D.C. proveniente de tres localidades de la región Ucayali. Rev Soc Quím Perú. 2014; 80(3): 174-182.

5. Poma W, Alcántara G. Estudio de suelos y capacidad de uso mayor del departamento de Cajamarca 2010-2011. [Internet]. Cajamarca: Gobierno Regional de Cajamarca. [Citado 22 may 2016]. Disponible en: http://zeeot.regioncajamarca.gob.pe/sites/dafault/ file/INFSUELOSZEE091.pdf. 
6. Agudelo I, Wagner HL, Gurni A, Ricco A. Dinámica de polifenoles y estudio anatomohistoquímico en Schinus longifolius (Lindl.) Speg. (Anacardiaceae) en respuesta a la infección por Calophya mammifex (Hemiptera - Calophyidae). B Latinoam Caribe Pl. 2013; 12(2):162-175.

7. AOAC. Official Methods of Analysis. Washington D.C.: Association Official Analitical Chemist Analytical Chemists; 2007.

8. Torres A. Caracterización física, química y compuestos bioactivos de pulpa de tomate de árbol (Cyphomandra betacea) (Cav.) Sendtn. Arch Latinoam Nutr. 2012; 62(4): 381388.

9. X- Rite. Guía para entender la comunicación del color. [Internet] 2012. [Citado 6 jul 2017]. Disponible en: http://www.mcolorcontrol.com/archivos/L10-001_Understand Color_es.pdf

10. Rojas BDS. Efecto de la estandarización y tratamiento térmico en el contenido de compuestos bioactivos y capacidad antioxidante en néctar de tomate de árbol (Solanum betaceum Cav.). [Tesis postgrado]. Lima, Perú: Universidad Nacional Agraria La Molina, Facultad de Industrias Alimentarias; 2013.

11. Belitz H, Grosch W, Schieberle P. Química de los Alimentos. $3^{\mathrm{a}}$ ed. Zaragoza: Editorial Acribia; 2011.

12. Barcelo J. Nicolas G. Sabates B, Sanchez R. Fisiologia vegetal. Madrid: Ediciones Piramide; 2009.

13. Chapoñan RMA, Medina VJI. Determinación del tiempo de vida útil de una salsa picante a partir de rocoto (Capsicum Pubescens) y tomate de árbol (Solamun betaceum). [Tesis]. Nuevo Chimbote, Perú: Universidad Nacional de El Santa, Facultad de Ingeniería; 2014. [Citado 3 ene 2017]. Disponible en: http://repositorio.uns.edu.pe/ handle/UNS/1947

14. Revelo VDA. Micro encapsulación de tomate de árbol rojo (Solanum betaceum Cav.) mediante spray drying para aplicación en productos lácteos. [Tesis postgrado]. Bogotá: Universidad Nacional de Colombia, Facultad de Ciencias Agrarias. 2014. [Citado 12 Jul 2017]. Disponible en: http://www.bdigital.unal.edu.co/46091/1/1113639059.2014.pdf

15. Soto M. Estudio fitoquímico de las hojas, flores y frutos de Solanum multifidum Lam. y Lycianthes lycioides (L.) Hassl. (Solanaceae) procedentes del Cerro Campana, Región La Libertad-Perú. Arnaldoa. 2014; 21(1): 91-104. 